\title{
A rara associação de dermatomiosite secundária à Chikungunya em fase crônica
}

\author{
The rare association of dermatomyositis secondary to Chikungunya in chronic phase \\ La rara asociación de dermatomiositis secundaria a Chikungunya en fase crónica
}

Recebido: 09/04/2021 | Revisado: 16/04/2021 | Aceito: 18/04/2021 | Publicado: 02/05/2021

\author{
Natacha Campos Arriaga \\ ORCID: https://orcid.org/0000-0002-1111-7090 \\ Hospital Geral Dr. Waldemar Alcântara, Brasil \\ E-mail: natachaarriaga@yahoo.com.br \\ Louise Cavalvanti Salles \\ ORCID: https://orcid.org/0000-0002-2704-1115 \\ Hospital Geral Dr. Waldemar Alcântara, Brasil \\ E-mail: louisecsalles@gmail.com \\ Francisco Theogenes Macedo Silva \\ ORCID: https://orcid.org/0000-0003-2290-4222 \\ Centro Universitário Christus, Brasil \\ E-mail: fcotheo@hotmail.com \\ Fabrício André Martins da Costa \\ ORCID: https://orcid.org/0000-0002-7080-1386 \\ Hospital Geral Dr. Waldemar Alcântara, Brasil \\ E-mail: fabricioam18@gmail.com \\ Cleonísio Leite Rodrigues \\ ORCID: https://orcid.org/0000-0003-0063-8971 \\ Hospital Geral de Fortaleza, Brasil \\ E-mail: cleonisiorodrigues@gmail.com \\ Kristopherson Lustosa Augusto \\ ORCID: https://orcid.org/0000-0001-9254-9129 \\ Universidade Federal do Ceará, Brasil \\ E-mail: kristopherson@hotmail.com
}

\begin{abstract}
Resumo
A chikungunya é uma doença tropical infecciosa causada pelo vírus Chikungunya (CHIKV), um vírus de RNA da família Togaviridae, gênero Alphavirus. É transmitida por mosquitos do gênero Aedes, infectados com o CHIKV. A doença evolui em três fases: aguda, subaguda e crônica, esta última tendo início após 3 meses de sintomatologia. A mialgia é um dos sintomas mais prevalentes na doença podendo ser intensa. Na literatura, alguns casos de Miopatia secundária à Chikungunya em fase aguda foram descritos, porém poucos foram relatados na fase crônica da doença. Apresentamos o caso de uma paciente feminina, que em 2016 desenvolveu fraqueza muscular progressiva às custas de dermatomiosite após dois anos da infecção pelo CHIKV.
\end{abstract}

Palavras-chave: Febre de chikungunya; Artralgia; Doenças autoimunes; Debilidade muscular.

\begin{abstract}
Chikungunya is an infectious tropical disease caused by Chikungunya virus (CHIKV), an RNA virus that belongs to the alphavirus genus of the family Togaviridae. It is a mosquito-borne viral disease transmitted by bites of Aedes aegypti and Aedes albopictus, most commonly. The disease evolves in three stages: acute, subacute and chronic. The chronic phase begins after 3 months of initial symptoms. Myalgia is one of the most prevalent symptoms in the disease and may be intense. In the literature, some Myopathy cases secondary to Chikungunya in acute phase were described, but few have been reported in the chronic disease. We present a case of a female patient, who developed progressive muscular weakness due to dermatomyositis in 2016 after 2 years of infection with CHIKV.

Keywords: Chikungunya fever; Arthralgia; Autoimmune diseases; Muscle weakness.

\section{Resumen}

El chikungunya es una enfermedad tropical infecciosa causada por el virus Chikungunya (CHIKV), un virus de ARN que pertenece al género alfavirus de la familia Togaviridae. Es una enfermedad viral transmitida por picaduras de Aedes aegypti y Aedes albopictus, más comúnmente. La enfermedad evoluciona en tres etapas: aguda, subaguda y crónica. La fase crónica comienza después de 3 meses de síntomas iniciales. La mialgia es uno de los síntomas más prevalentes de la enfermedad y puede ser intensa. En la literatura se describieron algunos casos de miopatía secundaria a Chikungunya en fase aguda, pero pocos se han reportado en la enfermedad crónica. Presentamos el caso de una paciente que desarrolló debilidad muscular progresiva por dermatomiositis en 2016 después de 2 años de infección por CHIKV.
\end{abstract}


Palabras clave: Fiebre chikungunya; Artralgia; Enfermidades autoinmunes; Debilidad muscular.

\section{Introdução}

Em setembro de 2014, o primeiro caso de Chikungunya foi detectado no Brasil, no estado do Amapá. No mesmo mês, um surto de CHIKV foi identificado na Bahia, em Feira de Santana. Em 2015, até a décima-quinta semana epidemiológica (de janeiro de 2015 a abril de 2015), 3.135 casos suspeitos foram notificados nos estados do Amapá e Bahia, sendo 1.688 confirmados: cinco por critérios laboratoriais e 1.683 por critérios clínicos-epidemiológicos (Brasil, 2015).

O Brasil vivenciou em 2015 a primeira epidemia de febre de Chikungunya. Até então, a doença era endêmica na África e no sudeste asiático, tendo ocorrido alguns surtos epidêmicos nestes locais nos últimos 60 anos (Rahim, Thekkekara, Bina \& Paul, 2016).

Neste período, diversos foram os desafios enfrentados pelos gestores em saúde pública para conter a replicação do mosquito transmissor, a fim de reduzir a sua propagação. As sequelas crônicas derivadas da doença pelo vírus CHIK parecem ser uma epidemia preocupante nas áreas endêmicas da América Latina. Estima-se que 25\% dos casos de doença pelo vírus evoluam com artralgia crônica (Rodríguez-Morales, Cardona-Ospina, Fernanda Urbano-Garzón \& Sebastian Hurtado-Zapata, 2016).

Há evidências que relacionam a doença causada pelo vírus CHIK com o desenvolvimento de artrite pós-viral inespecífica, artrite reumatóide, espondilites soronegativas e outros sintomas musculoesqueléticos não inflamatórios, como artralgia persistente (Mathew, Goyal, George, Thekkemuriyil, Jayakumar, Chopra \& Trivandrum, 2011). Essas sequelas têm afetado a qualidade de vida e aumentado a perda econômica direta e indireta (Cardona-Ospina, Villamil-Gómez, JimenezCanizales, Castañeda-Hernández \& Rodríguez-Morales, 2015) impondo uma carga significativa de doenças, com impactos consideráveis nos sistemas de saúde já sobrecarregados (Cardona-Ospina, Rodriguez-Morales \& Villamil-Gómez, 2015).

A febre de chikungunya pode afetar pessoas de todas as faixas etárias e sexo, desde que estejam expostas a áreas que tenham o mosquito transmissor. A doença é dividida didaticamente em três fases: aguda, subaguda e crônica. A fase aguda é caracterizada pelo início súbito de febre elevada, artralgia, mialgia e rash cutâneo. Outros sintomas que podem estar presentes são cefaleia, odinofagia, desconforto abdominal e constipação. Hiperemia conjuntival, conjuntivite persistente e linfadenopatia cervical também podem ocorrer.

A fase crônica ocorre quando, após 3 meses da infecção aguda, os pacientes persistem com sintomas articulares, mialgia e astenia.

Os pacientes afetados podem manifestar poliartrite inflamatória, tenossinovite ou bursite subaguda grave (consequentemente síndromes do túnel do nervo) nas mãos, punhos e exacerbação da dor ao movimento em articulações previamente lesadas. (Bandyopadhyay \& Ghosh, 2008)

A dermatomiosite (DM) é uma miopatia inflamatória idiopática (IIM) caracterizada por lesões cutâneas distintas e uma variedade de manifestações clínicas sistêmicas. Na ausência de achados dermatológicos característicos ou de miopatia, DM pode ser difícil de diagnosticar. Além disso, alguns estudos indicam que ela se manifesta, muitas vezes, como síndromes de "sobreposição", o que torna o seu diagnóstico ainda mais difícil. (DeWane, Waldman \& L, 2020)

O envolvimento da pele na DM geralmente se manifesta com pápulas características sobre os dedos, eritema nos cotovelos e joelhos, erupção cutânea em heliotrópio ao redor dos olhos, telangiectasias periungueais e distrofia cuticular (Callen \& Wortmann, 2006). Dentre os sintomas musculares, os principais são fraqueza muscular proximal, com ou sem mialgias ou perda de sensibilidade. Há uma associação bem estabelecida de DM com um risco aumentado de doenças neopláscias, manifestandi-se desse modo, algumas vezes, como sindrome paraneoplásica (Madan, Chinoy, Griffiths \& Cooper, 
2009). Outras características clínicas importantes do DM incluem a presença de doença pulmonar intersticial, aumentando assim a morbidade e mortalidade da doença.

Existem estudos clínicos que apoiam a hipótese de que doenças autoimunes possam ser desencadeadas por infecções, como lúpus eritematoso sistêmico (LES), síndrome anti-fosfolipídeo (SAF), síndrome de Sjögren, artrite reativa e esclerose múltipla (Zandman-Goddard \& Shoenfeld, 2005). Para algumas doenças autoimunes, algumas associações são bem descritas: Febre reumática e a infecção por estretococos do Grupo A, hepatite C e crioglobulinemia mista (Guilherme et al., 2000).

O presente estudo tem por objetivo descrever o caso de uma paciente feminina, que em 2016 desenvolveu fraqueza muscular progressiva às custas de dermatomiosite após dois anos da infecção pelo CHIKV.

\section{Metodologia}

\section{Tipo de Estudo}

Trata-se de um estudo descritivo de natureza qualitativa, do tipo estudo de caso, utilizando-se informações obtidas por meio da revisão do prontuário da paciente, com ênfase no acompanhamento durante o internamento hospitalar. O estudo de caso é uma investigação empírica que investiga um fenômeno contemporâneo dentro de seu contexto da vida real, especialmente quando os limites entre o fenômeno e o contexto não estão claramente definidos (Yin, 2015).

\section{Técnica e instrumento de coleta de dados}

Procedeu-se à revisão do prontuário para descrição do caso clínico e à revisão de literatura utilizando as bases científicas da National Library of Medicine (PubMed/MEDLINE), serviço online do National Center for Biothecnology Information - NCBI, EUA e Google Acadêmico.

\section{Aprovação do Comitê de Ética em Pesquisa}

Este trabalho foi submetido ao Comitê de Ética do Hospital Geral Dr. Waldemar Alcântara e aprovado em 08 de fevereiro de 2021.

\section{Caso Clínico}

Paciente feminina, 43 anos, natural do interior do estado do Ceará, apresentou-se à emergência médica em 2016 com queixa de exantema maculopapular não pruriginoso há aproximadamente dois anos, que surgiu após o diagnóstico clínico e laboratorial de Chikungunya (confirmado por Elisa, IgM positivo e IgG negativo), mais intenso no tórax e membros superiores, com piora progressiva. Refere ainda, associado ao quadro, artralgias, rubor, edema em ombros, cotovelos e punhos, sem rigidez matinal associada. Havia procurado atendimento em Unidade Básica de Saúde algumas vezes, onde fora prescrito glicocorticoide para controle dos sintomas. Fazia uso esporádico da medicação desde então, permanecendo sintomática e procurando atendimento médico regularmente. Duas semanas antes da admissão hospitalar, iniciou quadro de febre diária de aproximadamente $38^{\circ} \mathrm{C}$, piora do exantema maculopapular que se disseminou para o abdome e membros inferiores, rash malar com fotosenssibilidade, mialgia intensa e edema articular em punhos, joelhos e tornozelos, associado à fraqueza muscular proximal em membros inferiores.

Ao exame físico foi observado que a paciente apresentava fácies cushingoide, eritema em tronco e cervical posterior (sinal do xale), máculas de Gottron em cotovelos bilateralmente e máculas hipercrômicas e eritematosas difusas com descamação e livedo reticular em MMSS e MMII. O exame neurológico era normal, exceto por alteração na força muscular em MMII, que mostrava grau III/IV proximal, grau IV/V distal. A sensibilidade era preservada em MMII e reflexos normoativos. 
Foi realizada investigação clínica com solicitação de exames laboratoriais, cujo resultados evidenciaram anemia normocítica/normocrômica, leucocitose com linfocitose, transaminase glutâmico-ocalacética (TGO) 78U/L (valor de referência 5-40U/L), lactato desidrogenase (LDH) 723UI/L (valor de referência 120-246UI/L), velocidade de hemossedimentação (VHS) 45mm (valor de referência 5 -20mm), creatinofosfoquinase (CPK) 18,61U/L (valor de referência 33-211U/L) e aldolase 14,1U/L (valor de referência até 7,6U/L). Os valores do complemento mostraram-se dentro da normalidade (C3 152, C4 38 CH50 154,3). Foram ainda solicitados testes de autoimunidade, com anticoagulante lúpico, beta2-microglobulina, anticardiolipina, fator anti-nuclear (FAN), anti-histona, anti-RNP, anti-DNA dupla hélice, anti-SSa, anti-SSb, anti-SM, anti-Jo 1 e anti-Mi-2 não reagentes.

A Eletroneuromiografia (ENM), revelou atividade insercional aumentada, potenciais de fibrilação, ondas pontiagudas positivas, descargas espontâneas e alta frequência (Figura 1), além de potenciais de unidades motoras com amplitudes e duração reduzidas (Figura 2). Esses achados da ENM, embora não sejam específicos, corroboram o diagnóstico de miopatia inflamatória.

Figura 1: Eletroneuromiografia de músculo deltoide direito em repouso.

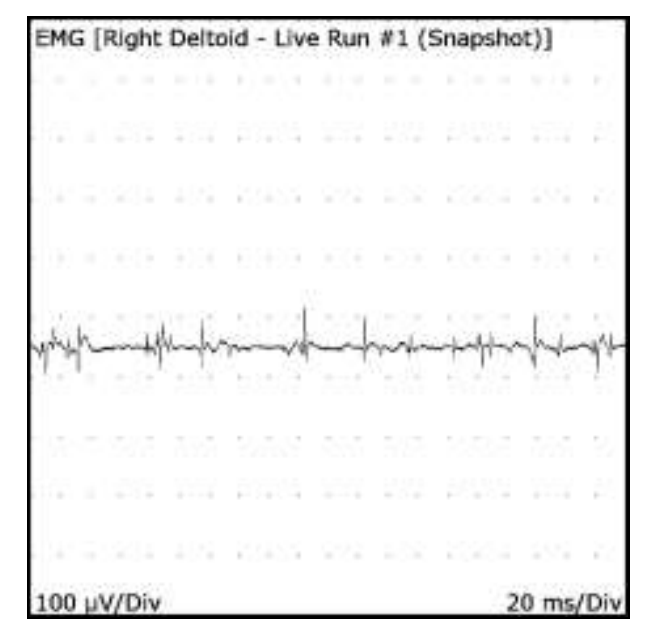

Fonte: Acervo individual Dr. Cleonísio Leite Rodrigues (2016).

Na Figura 1 observam-se potenciais de fibrilação e ondas pontiagudas positivas durante o estado de repouso em um músculo proximal, achado típico encontrado em pacientes portadores de miopatias inflamatórias. 
Figura 2: Eletroneuromiografia de músculo reto femoral durante contração.

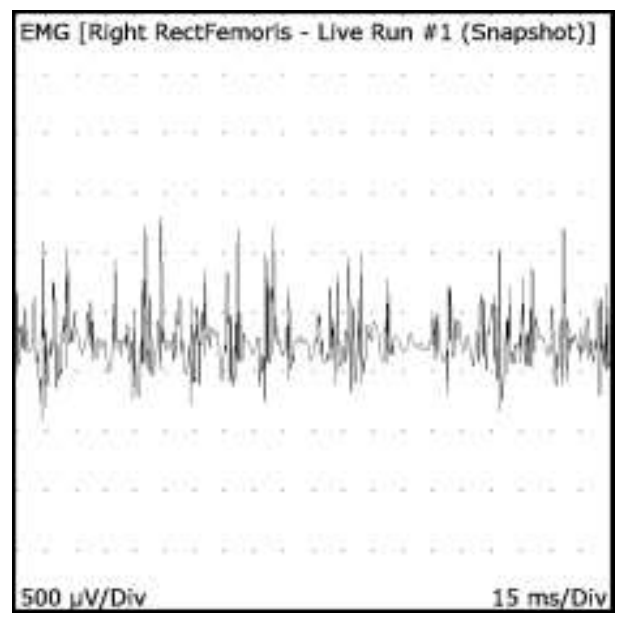

Fonte: Acervo individual Dr. Cleonísio Leite Rodrigues (2016).

Na Figura 2 observa-se um padrão de miopatia típico, com recrutamento precoce e pequenos e polifásicos potenciais de unidades durante a contração muscular ativa.

A pesquisa de neoplasias foi extensamente realizada com a realização de tomografias de tórax, abdome e pelve com contraste, colonoscopia, endoscopia digestiva alta, ultrassonografia transvaginal e de tireoide, e mamografia a fim de investigar dermatomiosite apresentando-se como síndrome paraneoplásica, porém nenhum exame mostrou alteração.

A paciente foi tratada com prednisona $1 \mathrm{mg} / \mathrm{kg} /$ dia com melhora dramática do quadro clínico e redução nos níveis de CPK, aldolase, TGO e LDH. Também foi iniciada azatioprina $3 \mathrm{mg} / \mathrm{kg} /$ dia, sendo esta medicação mantida após alta hospitalar. A paciente evoluiu com regressão dos sintomas articulares e melhora progressiva da força muscular. Segue atualmente em remissão do quadro clínico e com acompanhamento em ambulatório especializado de reumatologia.

\section{Discussão}

A Chikungunya é uma arbovirose causada pelo vírus Chikungunya (CHIKV), um arbovírus da família Togaviridae, gênero Alphavirus. É transmitida por mosquitos do gênero Aedes, infectados com o CHIKV. A doença evolui em três fases: aguda (primeiras 3 semanas), subaguda e crônica, esta última tendo início após 3 meses de sintomatologia. Na fase aguda, usualmente se manifesta por febre, poliartrite e mialgia, devendo sempre ser considerada como diagnóstico diferencial de poliartralgia febril (Cunha \& Trinta, 2017).

Desde o início da epidemia da doença, vários casos com acometimento neurológico também foram relatados, a maioria deles relacionados à encefalite (Agarwal, Vibha, Srivastava, Shukla, \& Prasad, 2017). O envolvimento do sistema nervoso periférico é menos frequente, assim como o desenvolvimento de miosite, esta última sendo relatada mais comumente na fase aguda da doença (Cunha \& Trinta, 2017).

As miopatias inflamatórias são ditas idiopáticas (MII) quando não se conseguem evidencias clínicas, laboratoriais e patológicas que identifiquem a etiologia da doença. Seus subtipos mais comuns são a dermatomiosite, a polimiosite e a miopatia por corpúsculos de inclusão. Elas possuem uma variedade de sintomas, dentre os principais se destacam a disfagia, fraqueza muscular, dor muscular e, em alguns casos mais graves, pneumopatia intersticial (Hochberg, 2018).

Assim como outras doenças autoimunes foram associadas a agentes infecciosos (Gan \& Miller, 2011) estudos sugerem que as infecções virais podem ser gatilhos para MII (Alouani, Fihmi, Ziz \& Dikhaye, 2017). 
Miopatias inflamatórias induzidas por drogas também já foram descritas. Em uma recente publicação sobre o assunto, as medicações mais associadas ao desenvolvimento de dermatomiosite foram a D-peicilamina, anti-inflamatórios não esteroidais (AINEs) e estatinas (Seidler \& Gottlieb 2008). Também há a descrição de um caso que relaciona o uso de doxiciclina ao desenvolvimento de dermatomiosite (Ren, Laumann, \& Silverberg, 2019).

Critérios foram propostos para avaliar a influência de agentes ambientais no desenvolvimento de doenças autoimunes, incluindo aqui a associação temporal, ausência de outras causas que justifiquem a doença, a regressão dos sintomas após a retirada do agente causador e a piora deles com a reexposição, plausibilidade biológica e resposta aos tratamentos instituídos (Miller et al., 2012). Dentre os agentes infecciosos virais, o Epstein-Barr vírus (EBV) foi o mais estudado, já sendo comprovado sua associação com dermatomiosite e polimiosite (Zheng, Zhu, Gao, Xu, \& Lu, 2019).

Existe também na literatura a associação entre miosite e infecção por CHIKV em um estudo que usou modelo com ratos, onde um clone molecular do vírus isolado do plasma humano foi inoculado na pata de ratos, produzindo uma resposta inflamatória importante, com histopatológico evidenciando miosite necrotizante, infiltrado inflamatório típico de artrite (histiócitos, neutrófilos, linfócitos e raras células plasmáticas), tenossinovite crônica e vasculite multifocal (Morrison et al., 2011).

A paciente do nosso relato foi infectada pelo vírus da chikungunya, há dois anos do aparecimento da fraqueza muscular, quando apresentou quadro de poliartrite simétricas de pequenas e grandes articulações, febre elevada e mialgia, época na qual ocorriam surtos da doença pelo Brasil. Nesta ocasião, o diagnóstico da doença havia sido realizado com base em critérios clínicos e epidemiológicos.

Durante o internamento, foi confirmada a exposição prévia da paciente ao vírus e iniciada a investigação para outros diagnósticos diferenciais de fraqueza muscular. Foram excluídas doenças autoimunes primárias e secundárias a doenças neoplásicas (síndrome paraneoplásica) e realizado exames complementares. A eletroneuromiografia sugeriu padrão típico de miopatia inflamatória (atividade insercional aumentada, ondas pontiagudas positivas, descargas espontâneas e alta frequência e potenciais de unidades motoras com amplitudes e duração reduzidas), sendo esses achados comuns na dermatomiosite (Blijham, Hengstman, Hama-Amin, van Engelen \& Zwarts, 2006).

\section{Conclusão}

Descrevemos, portanto, o primeiro relato nacional da associação entre dermatomiosite e chikungunya em fase crônica, devendo a infecção viral ter funcionado como trigger para o desenvolvimento de autoimunidade. Sugere-se, portanto, que mais pesquisas sejam realizadas continuamente na área clínica para consolidar esse agente causal como trigger da doença e sua etiopatogenia em humanos.

\section{Referências}

Agarwal, A., Vibha, D., Srivastava, A. K., Shukla, G., \& Prasad, K. (2017). Guillain-Barre syndrome complicating chikungunya virus infection. Journal of neurovirology, 23(3), 504-507. https://doi.org/10.1007/s13365-017-0516-1

Alouani, I., Fihmi, N., Zizi, N., \& Dikhaye, S. (2017). Dermatomyositis revealing both a metastatic linitis plastica and hepatitis C virus infection. Indian journal of dermatology, venereology and leprology, 83(5), 606-609. https://doi.org/10.4103/ijdvl.IJDVL_697_16

Bandyopadhyay, D., \& Ghosh, S. K. (2008). Mucocutaneous features of Chikungunya fever: a study from an outbreak in West Bengal, India. International journal of dermatology, 47(11), 1148-1152. https://doi.org/10.1111/j.1365-4632.2008.03817.x

Blijham, P. J., Hengstman, G. J., Hama-Amin, A. D., van Engelen, B. G., \& Zwarts, M. J. (2006). Needle electromyographic findings in 98 patients with myositis. European neurology, 55(4), 183-188. https://doi.org/10.1159/000093866

Brasil. (2015). Monitoramento dos casos de dengue e febre de chikungunya até a Semana Epidemiológica 15. Ministério da Saúde. http://portalsaude.saude.gov.br/images/pdf/2015/maio/04/2015-016---Boletim-Dengue-SE15-2015.pdf.

Callen, J. P., \& Wortmann, R. L. Dermatomyositis. Clin Dermatol. 24(5):363-73. 10.1016/j.clindermatol.2006.07.001. PMID: 16966018. 
Cardona-Ospina, J. A., Rodriguez-Morales, A. J., \& Villamil-Gómez, W. E. (2015). The burden of Chikungunya in one coastal department of Colombia (Sucre): Estimates of the disability adjusted life years (DALY) lost in the 2014 epidemic. Journal of infection and public health, 8(6), 644-646. https://doi.org/10.1016/j.jiph.2015.06.001

Cardona-Ospina, J. A., Villamil-Gómez, W. E., Jimenez-Canizales, C. E., Castañeda-Hernández, D. M., \& Rodríguez-Morales, A. J. (2015). Estimating the burden of disease and the economic cost attributable to chikungunya, Transactions of the Royal Society of Tropical Medicine and Hygiene, 109(12), 793-802. https://doi.org/10.1093/trstmh/trv094

Cunha, R. V. da., \& Trinta, K. S. (2017). Chikungunya virus: clinical aspects and treatment - A Review. Memórias do Instituto Oswaldo Cruz, 112(8), 523531. https://doi.org/10.1590/0074-02760170044

DeWane, M. E., Waldman, R., \& Lu, J. (2020). Dermatomyositis: Clinical features and pathogenesis. Journal of the American Academy of Dermatology, 82(2), 267-281. https://doi.org/10.1016/j.jaad.2019.06.1309

Gan, L., \& Miller, F. W. (2011). State of the art: what we know about infectious agents and myositis. Current opinion in rheumatology, 23(6), 585-594. https://doi.org/10.1097/BOR.0b013e32834b5457

Guilherme, L., Dulphy, N., Douay, C., Coelho, V., Cunha-Neto, E., Oshiro, S. E., Assis, R. V., Tanaka, A. C., Pomerantzeff, P. M., Charron, D., Toubert, A., \& Kalil, J. (2000). Molecular evidence for antigen-driven immune responses in cardiac lesions of rheumatic heart disease patients. International immunology, 12(7), 1063-1074. https://doi.org/10.1093/intimm/12.7.1063

Madan, V., Chinoy, H., Griffiths, C. E., \& Cooper, R. G. (2009). Defining cancer risk in dermatomyositis. Part I. Clinical and experimental dermatology, 34(4), 451-455. https://doi.org/10.1111/j.1365-2230.2009.03216.x

Marc Hochberg, M. R. (2018). Rheumatology. (7a ed.). Elsevier.

Mathew, A. J., Goyal, V., George, E., Thekkemuriyil, D. V., Jayakumar, B., Chopra, A., \& Trivandrum COPCORD Study Group (2011). Rheumaticmusculoskeletal pain and disorders in a naïve group of individuals 15 months following a Chikungunya viral epidemic in south India: a population based observational study. International journal of clinical practice, 65(12), 1306-1312. https://doi.org/10.1111/j.1742-1241.2011.02792.x

Miller, F. W., Pollard, K. M., Parks, C. G., Germolec, D. R., Leung, P. S., Selmi, C., Humble, M. C., \& Rose, N. R. (2012). Criteria for environmentally associated autoimmune diseases. Journal of autoimmunity, 39(4), 253-258. https://doi.org/10.1016/j.jaut.2012.05.001

Morrison, T. E., Oko, L., Montgomery, S. A., Whitmore, A. C., Lotstein, A. R., Gunn, B. M., Elmore, S. A., \& Heise, M. T. (2011). A mouse model of chikungunya virus-induced musculoskeletal inflammatory disease: evidence of arthritis, tenosynovitis, myositis, and persistence. The American journal of pathology, 178(1), 32-40. https://doi.org/10.1016/j.ajpath.2010.11.018

Rahim, A. A., Thekkekara, R. J., Bina, T., \& Paul, B. J. (2016). Disability with Persistent Pain Following an Epidemic of Chikungunya in Rural South India. The Journal of rheumatology, 43(2), 440-444. https://doi.org/10.3899/jrheum.141609

Ren, Z., Laumann, A. E., \& Silverberg, J. I. (2019). Association of dermatomyositis with systemic and opportunistic infections in the United States. Archives of dermatological research, 311(5), 377-387. https://doi.org/10.1007/s00403-019-01913-0

Rodríguez-Morales, A. J., Cardona-Ospina, J. A., Fernanda Urbano-Garzón, S., \& Sebastian Hurtado-Zapata, J. (2016). Prevalence of Post-Chikungunya Infection Chronic Inflammatory Arthritis: A Systematic Review and Meta-Analysis. Arthritis care \& research,68(12), 1849-1858. https://doi.org/10.1002/acr.22900

Seidler, A. M., \& Gottlieb, A. B. (2008). Dermatomyositis induced by drug therapy: a review of case reports. Journal of the American Academy of Dermatology, 59(5), 872-880. https://doi.org/10.1016/j.jaad.2008.05.034

Yin, R. K. (2015). Estudo de caso: planejamento e métodos (5ª ed.). (C. M. Herrera, Trad.): Bookman. (Obra original publicada em 1984).

Zandman-Goddard, G., \& Shoenfeld, Y. (2005). Infections and SLE. Autoimmunity, 38(7), 473-485. https://doi.org/10.1080/08916930500285352

Zheng, Q., Zhu, K., Gao, C. N., Xu, Y. P., \& Lu, M. P. (2019). Prevalence of Epstein-Barr virus infection and characteristics of lymphocyte subsets in newly onset juvenile dermatomyositis. World journal of pediatrics: WJP, 10.1007/s12519-019-00314-7. Advance online publication. https://doi.org/10.1007/s12519019-00314-7 